\title{
Extracellular Vesicles - powerful markers of cancer EVolution
}

\author{
Joana Carvalho ${ }^{*}$ and Carla Oliveira ${ }^{1,2}$ \\ 1 Institute of Molecular Pathology and Immunology of University of Porto (IPATIMUP), Porto, Portugal \\ 2 Department of Pathology and Oncology, Faculty of Medicine of University of Porto, Porto, Portugal \\ ${ }^{*}$ Correspondence: jcarvalho@ipatimup.pt
}

Edited by:

Matías Sáenz-Cuesta, Biodonostia Health Research Institute, Spain

Reviewed by:

Miroslaw Kornek, University of Saarland, Germany

Olga Shatneva, University Hospital Cologne, Germany

Keywords: extracellular vesicles, longitudinal studies, precision-medicine, cancer biomarkers, liquid biopsy

Solid tumors are likely derived from the coevolution of neoplastic cells, stromal components, vasculature, and immune cells (1). As tumors evolve and progress, an array of molecular changes accumulate giving rise to multiple cell subpopulations, each with the ability to divide and mutate further. In addition, neoplastic cell populations are able to modulate the behavior of other types of cells in their microenvironment, converting their intrinsic antitumoral into pro-tumoral activity $(2,3)$. Therefore, a malignant tumor is composed not only of neoplastic cells that are heterogeneous in terms of genetic and phenotypic features, but also by different protumoral cells and a particular extracellular matrix that supports cancer evolution and progression.

Tumor heterogeneity has been recognized as one of the main factors for cancer therapy failure, and has just started to be dissected using next-generation sequencing (NGS) approaches (4). While whole genome sequencing, and particularly, exome sequencing have provided the molecular basis for several complex traits, RNA and bisulfite sequencing have been important to disclose expression regulatory mechanisms. However, NGS-derived studies have often been conducted using single fragments/biopsies of primary tumors, and therefore fail to reflect the global tumor heterogeneity, dynamics, and drug sensitivities, likely to change during tumor evolution and treatment. For these reasons, there is the need to develop strategies that may accurately capture the entire landscape and allow following clonal evolution of tumor populations.
Scientific evidence supports that most types of cells secrete small vesicles (exosomes and microvesicles) into the extracellular milieu (5), and that tumor cells in particular produce at least threefold more of these small vesicles than normal cells (6-8). These so-called extracellular vesicles (EVs) are emerging mediators of intercellular communication and orchestrators of health and disease, and contain a repertoire of genetic information (incorporated in DNA, RNA, microRNAs, and proteins), which may be a fingerprint of the releasing cell type $(9,10)$. EVs can be easily detected in biological fluids such as plasma, serum, ascites, or urine, and provide excellent minimally invasive biomarker candidates to monitor cancer patients' progression, prognosis, and treatment efficacy (10, 11). In fact, tumor-derived exosomes in patients' bloodstream were shown to contain fractions of tumor genome, transcriptome, and proteome such as KRAS, TP53 mutations in pancreatic and colon cancer (12); mutant/variant EGFRvIII mRNAs in glioblastoma (13); microRNAs in ovarian cancer (14); MET in melanoma (15); and HER2 in breast cancer (16). Further, double-stranded DNA (exoDNA) representing the entire genome and reflecting the mutational status of parental tumor cells [e.g., $B R A F(\mathrm{~V} 600 \mathrm{E})$ and EGFR exon 19 deletion - del19], was found in EVs from melanoma and non-small cell lung cancer cell lines (17). From a clinical perspective, molecules enclosed in EVs harbor potential usefulness as circulating biomarkers with impact in early detection and during cancer progression. Apart from carrying specific molecular signatures and disease effectors, EVs also contribute to horizontal cellular transformation and phenotypic reprograming, both locally and systemically $(8,10,15,18)$.

The identification of specific EV features has allowed developing isolation and characterization methodologies that have been used in numerous studies (19-21). Most of these have been focused on the characterization of the cargo of EVs in different types of cancer, using either conditioned media of cancer cell lines, or unique samples from cancer patients' body fluids. Cancer cell line studies have provided markers of EVs for different types of cancers, however, lack the representativeness of cancer as a heterogeneous cell population. Unique samples from cancer patients' body fluids have highlighted potential markers for cancer diagnosis and prognosis in crosssectional studies, although fail to deliver useful information to monitor tumor heterogeneity and dynamics, and to allow therapy response and recurrence assessment.

Longitudinal studies of cancer patients, from whom samples are repeatedly collected along diagnosis, treatment, and follow-up, have been rarely reported, and may be the most adequate tools to address the abovementioned limitations. One possible longitudinal approach should enclose the molecular profiling of the following patient-derived samples: (1) biopsy/surgical tumor specimens; (2) body fluid-derived EVs collected prior/at surgery; (3) body fluid-derived EVs collected immediately after surgery; (4) body fluid-derived EVs collected along therapy cycles, and; (5) body fluid-derived EVs collected after disease remission (if possible). 
The comparative analysis of data derived from each of these datasets will shed light into cancer-specific signatures that become represented in tumor-derived EVs (biomarker candidates), and that should be used to monitor tumor evolution, dynamics, and therapy response, as well as predict disease recurrence. This type of studies raises the need for close collaborations between clinical and basic research teams, to set-up effective study designs and ethically approved protocols, to allow collection of multiple samples and clinically relevant information from each individual patient. The power of this approach is the possibility of providing information useful for the design of precision-medicine approaches, with impact in clinical practice.

In summary, in this article, we discuss the impact of performing longitudinal studies through the analysis of EVs from cancer patients, to improve our understanding of tumor heterogeneity/evolution, and to identify minimally invasive markers, potentially useful for disease management of cancer patients. We further present a workflow that may be useful to consider when designing longitudinal studies involving cancer patients. Despite lengthy and labor-intensive, such studies will certainly provide answers for currently unsolved questions in cancer research.

At this point, it is clear that EVs have a tremendous potential to be used as a "liquid biopsy" for cancer patients, which would be less invasive compared to surgery and may provide diagnostic information, aid in therapeutic decisions, and monitoring of disease over time, on a personalized basis.

\section{ACKNOWLEDGMENTS}

Authors would like to acknowledge the COST Action BM1202 European Network On Microvesicles And Exosomes In Health And Disease (Me-Had) and the Portuguese Foundation for Science and Technology (FCT) for funding Postdoc fellowship to Joana Carvalho (ref: SFRH/BPD/86543/2012). The fellowship has been funded in the frame of "Programa Operacional Temático Factores de
Competitividade (COMPETE) e comparticipado pelo fundo Comunitário Europeu FEDER.”

\section{REFERENCES}

1. Junttila MR, de Sauvage FJ. Influence of tumour micro-environment heterogeneity on therapeutic response. Nature (2013) 501(7467):346-54. doi: $10.1038 /$ nature 12626

2. Holzel M, Bovier A, Tuting T. Plasticity of tumour and immune cells: a source of heterogeneity and a cause for therapy resistance? Nat Rev Cancer (2013) 13(5):365-76. doi:10.1038/nrc3498

3. Acharyya S, Oskarsson T, Vanharanta S, Malladi S, Kim J, Morris PG, et al. A CXCL1 paracrine network links cancer chemoresistance and metastasis. Cell (2012) 150(1):165-78. doi:10.1016/j.cell.2012. 04.042

4. Renovanz M, Kim EL. Intratumoral heterogeneity, its contribution to therapy resistance and methodological caveats to assessment. Front Oncol (2014) 4:142. doi:10.3389/fonc. 2014.00142

5. Raposo G, Stoorvogel W. Extracellular vesicles: exosomes, microvesicles, and friends. J Cell Biol (2013) 200(4):373-83. doi:10.1083/jcb.201211138

6. van Doormaal FF, Kleinjan A, Di Nisio M, Buller HR, Nieuwland R. Cell-derived microvesicles and cancer. Neth J Med (2009) 67(7):266-73.

7. Rabinowits G, Gercel-Taylor C, Day JM, Taylor DD, Kloecker GH. Exosomal microRNA: a diagnostic marker for lung cancer. Clin Lung Cancer (2009) 10(1):42-6. doi:10.3816/CLC.2009.n.006

8. Melo SA, Sugimoto H, O'Connell JT, Kato N, Villanueva A, Vidal A, et al. Cancer exosomes perform cell-independent microRNA biogenesis and promote tumorigenesis. Cancer Cell (2014) 26(5):707-21. doi:10.1016/j.ccell.2014.09.005

9. Valadi H, Ekstrom K, Bossios A, Sjostrand M, Lee JJ, Lotvall JO. Exosome-mediated transfer of mRNAs and microRNAs is a novel mechanism of genetic exchange between cells. Nat Cell Biol (2007) 9(6):654-9. doi:10.1038/ncb1596

10. Julich H, Willms A, Lukacs-Kornek V, Kornek M. Extracellular vesicle profiling and their use as potential disease specific biomarker. Front Immunol (2014) 5:413. doi:10.3389/fimmu.2014. 00413

11. Rak J. Extracellular vesicles - biomarkers and effectors of the cellular interactome in cancer. Front Pharmacol (2013) 4:21. doi:10.3389/fphar.2013. 00021

12. Kahlert C, Melo SA, Protopopov A, Tang J, Seth $\mathrm{S}$, Koch M, et al. Identification of double-stranded genomic DNA spanning all chromosomes with mutated KRAS and p53 DNA in the serum exosomes of patients with pancreatic cancer. J Biol Chem (2014) 289(7):3869-75. doi:10.1074/ jbc.C113.532267

13. Skog J, Wurdinger T, van Rijn S, Meijer DH, Gainche L, Sena-Esteves M, et al. Glioblastoma microvesicles transport RNA and proteins that promote tumour growth and provide diagnostic biomarkers. Nat Cell Biol (2008) 10(12):1470-6. doi: $10.1038 /$ ncb 1800
14. Taylor DD, Gercel-Taylor C. MicroRNA signatures of tumor-derived exosomes as diagnostic biomarkers of ovarian cancer. Gynecol Oncol (2008) 110(1):13-21. doi:10.1016/j.ygyno.2008.04.033

15. Peinado H, Aleckovic M, Lavotshkin S, Matei I, Costa-Silva B, Moreno-Bueno G, et al. Melanoma exosomes educate bone marrow progenitor cells toward a pro-metastatic phenotype through MET. Nat Med (2012) 18(6):883-91. doi:10.1038/nm. 2753

16. Ciravolo V, Huber V, Ghedini GC, Venturelli E, Bianchi F, Campiglio $\mathrm{M}$, et al. Potential role of HER2-overexpressing exosomes in countering trastuzumab-based therapy. J Cell Physiol (2012) 227(2):658-67. doi:10.1002/jcp.22773

17. Thakur BK, Zhang H, Becker A, Matei I, Huang Y, Costa-Silva B, et al. Double-stranded DNA in exosomes: a novel biomarker in cancer detection. Cell Res (2014) 24(6):766-9. doi:10.1038/cr.2014.44

18. Demory Beckler M, Higginbotham JN, Franklin JL, Ham AJ, Halvey PJ, Imasuen IE, et al. Proteomic analysis of exosomes from mutant KRAS colon cancer cells identifies intercellular transfer of mutant KRAS. Mol Cell Proteomics (2013) 12(2):343-55. doi:10.1074/mcp.M112.022806

19. Dragovic RA, Gardiner C, Brooks AS, Tannetta DS, Ferguson DJ, Hole P, et al. Sizing and phenotyping of cellular vesicles using nanoparticle tracking analysis. Nanomedicine (2011) 7(6):780-8. doi:10. 1016/j.nano.2011.04.003

20. van der Vlist EJ, Nolte-'t Hoen EN, Stoorvogel W, Arkesteijn GJ, Wauben MH. Fluorescent labeling of nano-sized vesicles released by cells and subsequent quantitative and qualitative analysis by high-resolution flow cytometry. Nat Protoc (2012) 7(7):1311-26. doi:10.1038/nprot.2012.065

21. Van Deun J, Mestdagh P, Sormunen R, Cocquyt V, Vermaelen K, Vandesompele J, et al. The impact of disparate isolation methods for extracellular vesicles on downstream RNA profiling. J Extracell Vesicles (2014) 3:24858. doi:10.3402/jev.v3.24858

Conflict of Interest Statement: The authors declare that the research was conducted in the absence of any commercial or financial relationships that could be construed as a potential conflict of interest.

Received: 28 November 2014; paper pending published: 05 December 2014; accepted: 19 December 2014; published online: 12 January 2015.

Citation: Carvalho J and Oliveira C (2015) Extracellular Vesicles - powerful markers of cancer EVolution. Front. Immunol. 5:685. doi: 10.3389/fimmu.2014.00685 This article was submitted to Inflammation, a section of the journal Frontiers in Immunology.

Copyright (c) 2015 Carvalho and Oliveira. This is an open-access article distributed under the terms of the Creative Commons Attribution License (CC BY). The use, distribution or reproduction in other forums is permitted, provided the original author(s) or licensor are credited and that the original publication in this journal is cited, in accordance with accepted academic practice. No use, distribution or reproduction is permitted which does not comply with these terms. 